\title{
DERMATOFITOSIS PODAL EN PACIENTES DIABETICOS TIPO 1 y 2
}

\author{
(Podal dermatophytosis in diabetic patients tipe 1 and 2)
}

\author{
Maria Cristina Díaz ${ }^{(1)}$ Perla Awad ${ }^{(2)}$ \\ Hector Cabrera ${ }^{(3)}$, Gladys Yentzen M. ${ }^{(4)}$ \\ 1.- Programa de Microbiología y Micología, ICBM, Facultad de Medicina, Universidad de Chile, \\ Independencia 1027, Santiago, mcdiaz@med.uchile.cl \\ 2.- Clínica Avansalud, 3.- Alumno $5^{\circ}$ año Escuela Tecnología Médica, Universidad de Chile. \\ 4.- Escuela de Salud Pública, Facultad de Medicina, Universidad de Chile
}

Palabras clave: Diabetes, dermatofitosis

Key words: Diabetes, dermatophytosis

\section{RESUMEN}

La diabetes mellitus (DM) es un síndrome metabólico caracterizado por hiperglicemia. Los pacientes diabéticos presentan una mayor predisposición a las infecciones fúngicas superficiales y los agentes involucrados con mayor frecuencia corresponden a Candida spp. y dermatofitos (Trichophyton rubrum, T. mentagrophytes, entre otros). En la presente investigación se determinó la frecuencia de dermatofitos en los pies de pacientes diabéticos tipo 1 y 2 relacionándolos con los factores epidemiológicos asociados. Entre Marzo y Junio 2006 , se examinaron los pies a 164 pacientes (75 mujeres y 89 hombres ) entre 29 y 84 años, que acudieron a la Asociación de Diabéticos de Chile (ADICH) para su control de rutina. Se recolectaron 202 muestras clínicas (30 interortejos, 17 plantar y 155 uñas pies), que se cultivaron en Agar Sabouraud Glucosado y Lactritmel a $25^{\circ}$ y $37^{\circ} \mathrm{C}$ por un período de 21 días. La mayoría de los pacientes eran DM Tipo 2 (79\%). En 50/164 pacientes se obtuvo cultivo positivo, de 202 muestras clínicas analizadas se aisló dermatofitos en un $27.7 \%$. T. rubrum fue el dermatofito con mayor aislamiento en pacientes DM 1 y 2 con cifras cercanas al 80\%, mientras T. mentagrophytes fue aparentemente superior en DM 2. T. rubrum obtuvo las mayores frecuencias en todos los tipos de muestras (DM 1 y DM 2), mientras, T. mentagrophytes también se presentó en todos los tipos de muestras de DM 2 y solo en uñas en pacientes DM 1. Los hallazgos micológicos en los pies de pacientes diabéticos son similares a lo reportado en la literatura.

Recibido: 15 de Octubre 2007

Aceptado: 11 de Diciembre 2007

\begin{abstract}
The mellitus diabetes (DM) is a metabolic syndrome which is characterized by hyperglykemia. Diabetic patients are very much liable to suffer from superficial fungal infections and involved agents occurring more frequently are the following: Candida spp and dermatophytes (Trichophyton rubrum, T.mentagrophytes, among others).

It was concluded in the present research work the frequency of dermatophytes in the feet of type 1 and 2 diabetic patients, by relating them with the associated epidemiological factors. Within march and june 2006, feet of 29 to 84-aged 164 patients (75 female and 89 male) who attended the Asociación de Diabéticos de Chile (ADICH) to get their routine control, were examined. Two hundred and two clinical samples were collected (30 interortejos, 17 plantar and 155 toe nails) which were cultured in Sabouraud Glucose Agar and Lactrimel at $25^{\circ}$ and $37^{\circ} \mathrm{C}$ for a 21-day period.

Most patients wereType 2 DM (79\%). In 50/164 patients culture resulted to be positive, out of 202 clinical samples analyzed, dermatophytes were $27,7 \%$ isolated. T.rubrum turned to be the dermatophyte with the highest isolation in DM 1 and 2 patients, yielding numbers as high as $80 \%$, whereas T.mentagrophytes was apparently 27,7\% greater in DM 2 patients. T.rubrum reached the highest frequencies in all kinds of samples (DM 1 and $D M$ 2), while T.mentagrophytes was also present in all the DM 2 samples and only in the nails of DM 1 patients.

Mycological findings in the feet of diabetic patients are similar to those cited in literature.
\end{abstract}




\section{INTRODUCCION}

La diabetes mellitus (DM) es un síndrome metabólico caracterizado por hiperglicemia debida a la secreción o acción de la insulina y según la nueva clasificación, ADA 1997 y OMS 1999 se propone utilizar las designaciones DM tipo 1y tipo 2, gestacional y otros tipos específicos (National Diabetes Data Group). La primera se caracteriza por daño de las células â pancreáticas con hiposecreción absoluta de insulina, manifestándose con tendencia a la cetosis, mientras que en la segunda, que es la más frecuente, están aquellos pacientes en los cuales coexiste insulinoresistencia y niveles insuficientes de insulina. Clínicamente, la mayoría de estos últimos individuos son obesos o con aumento de la grasa abdominal, metabólicamente estables, habitualmente no requieren tratamiento insulínico para vivir y tienen una evolución subclínica durante largo tiempo; llegan a requerir terapia con insulina después de años de evolución por fracaso secundario a las drogas orales (Harrison, 2006).

Se ha observado que el número de individuos diagnosticados con esta enfermedad metabólica está en aumento, afectando a toda la población y a diferentes grupos etáreos. Actualmente 190 millones de personas padecen de DM en el mundo y se calcula que las cifras ascenderían a 330 millones en 2025 (Harrison, 2006). En el año 2000, OPS ha señalado que en Latinoamerica y el Caribe 19 millones presentaban DM y alcanzaría a 40 millones en el 2025. En USA es un importante problema de salud pública y en Chile afecta al 5-10\% de la población (García de Los Ríos, 1992). Las infecciones bacterianas y fúngicas en la DM por lo general se encuentran localizadas en la piel y se relacionan con la glicemia y el control metabólico, la frecuencia de onicomicosis es más elevada que en la población general especialmente en pacientes sin control de su glicemia y existe controversia entre la frecuencia de onicomicosis y el adecuado control metabólico (Grupta et al., 1998; Dogra et al., 2002; Bougerra, et al., 2004; Mayser et al., 2004; Mlinaric-Missoni et al., 2005).

Dado que en nuestro país existe una escasa información al respecto, el propósito de este estudio fue determinar la frecuencia de dermatofitosis en pies de pacientes diabéticos tipo 1 y 2 y relacionar estos hallazgos con factores epidemiológicos asociados, para que sirva como antecedentes en la aplicación de medidas preventivas.

\section{MATERIALES Y METODOS}

El diseño del estudio es transversal descriptivo con un tipo de muestreo no probabilístico, por conveniencia. Entre Marzo y Junio del 2006, se examinaron los pies a 164 pacientes diabéticos que acudieron a su control de rutina en un centro de diabetes (Asociación de Diabetes de Chile-ADICH).

Los individuos seleccionados fueron informados del estudio; a cada uno de ellos se les aplicó una encuesta en relación a identificación del paciente, edad, sexo, tipo de diabetes y existencia de lesiones compatibles clínicamente con una micosis.

Todo paciente que se encontraba con tratamiento antifúngico tópico u oral, embarazadas o lactando u otro tipo de inmunosupresión fueron excluidos del estudio .

Del total de pacientes se obtuvo 202 muestras clínicas distribuidas en: 30 escamas de interortejos, 17 plantares y 155 uñas de pies. En cada muestra se realizó un cultivo en Agar Sabouraud Glucosado al 2\% y Lactritmel, ambos adicionados con Cloranfenicol ( $250 \mathrm{mg} / \mathrm{L}$ ) e incubados a $25^{\circ}$ y $37^{\circ} \mathrm{C}$ por un período de 21 días.

La identificación de los hongos aislados se basó en sus características macro y micromorfológicas (De Hoog, et al., 2000).

Tabla 1: Distribución de los pacientes diabéticos consultantes en ADICH según tipo de diabetes en relación a edad y sexo

\begin{tabular}{|c|c|c|c|c|c|c|c|c|c|c|}
\hline \multirow{3}{*}{ EDAD } & \multicolumn{3}{|c|}{ HOMBRES } & \multicolumn{4}{|c|}{ MUJERES } & \multicolumn{3}{|c|}{ TOTALES } \\
\hline & DM tipo 1 & DM tipo 2 & \multirow{2}{*}{$\begin{array}{r}\text { Total } \\
\mathbf{N}^{\circ}\end{array}$} & DM tipo 1 & \multicolumn{2}{|c|}{ DM tipo 2} & \multirow{2}{*}{$\begin{array}{c}\text { Total } \\
\mathbf{N}^{\circ}\end{array}$} & DM tipo1 & DM tipo 2 & \multirow{2}{*}{$\begin{array}{l}\text { Total } \\
\mathbf{N}^{\mathbf{0}}\end{array}$} \\
\hline & $\mathrm{N}^{\circ} \quad \%$ & $\mathrm{~N}^{\mathrm{o}} \quad \%$ & & $\mathrm{~N}^{\circ} \quad \%$ & $\mathbf{N}^{\circ}$ & $\%$ & & $\mathrm{~N}^{\circ} \quad \%$ & $\mathbf{N}^{\circ}$ & \\
\hline$<40$ & $\begin{array}{ll}2 & 50\end{array}$ & $\begin{array}{ll}2 & 50\end{array}$ & 4 & 0 & & - & 0 & $\begin{array}{ll}2 & 50\end{array}$ & $\begin{array}{ll}2 & 50\end{array}$ & 4 \\
\hline $40-49$ & 0 & $\begin{array}{ll}8 & 100\end{array}$ & 8 & 0 & 3 & 100 & 3 & - & $11 \quad 100$ & 11 \\
\hline $50-59$ & 12 & $22 \quad 88$ & 25 & $\begin{array}{ll}4 & 18\end{array}$ & 18 & 82 & 22 & $\begin{array}{ll}7 & 15\end{array}$ & 40 & 47 \\
\hline $60-69$ & 7 & $\begin{array}{ll}25 & 93\end{array}$ & 27 & $\begin{array}{ll}6 & 24\end{array}$ & 19 & 76 & 25 & $\begin{array}{ll}8 & 15\end{array}$ & $\begin{array}{ll}44 & 85\end{array}$ & 52 \\
\hline $70-79$ & $\begin{array}{ll}4 & 17\end{array}$ & 1983 & 23 & $\begin{array}{ll}4 & 21\end{array}$ & 15 & 79 & 19 & $\begin{array}{ll}8 & 19\end{array}$ & 34 & 42 \\
\hline$>80$ & 0. & $\begin{array}{ll}2 & 100\end{array}$ & 2 & $\begin{array}{ll}1 & 17\end{array}$ & 5 & 83 & 6 & 4 & 88 & 8 \\
\hline TOTAL & $11 \quad 12$ & $\begin{array}{ll}78 & 88\end{array}$ & 89 & $15 \quad 20$ & 60 & 80 & 75 & $26 \quad 16$ & $138 \quad 84$ & 164 \\
\hline
\end{tabular}


Tabla 2.- Resultados del cultivo micológico en 202 muestras clínicas analizadas en 164 pacientes diabéticos.

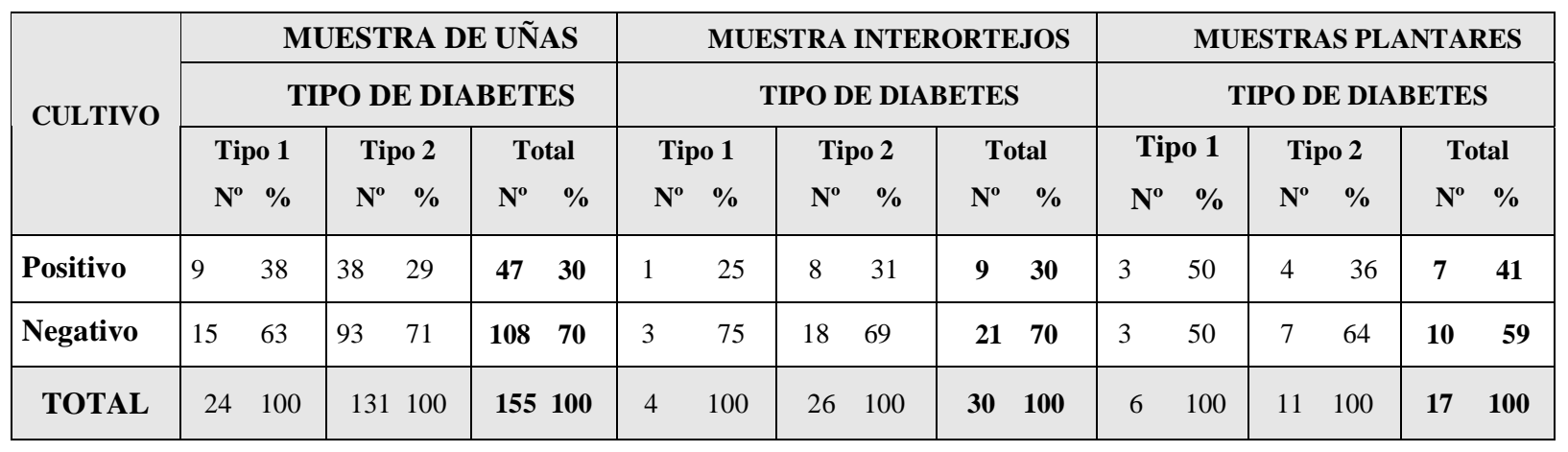

\section{RESULTADOS}

De los 164 pacientes estudiados , 89 eran del sexo masculino (54 \%) y 75 del sexo femenino (46\%), cuyos rangos de edad variaron entre 28 y 84 años y una mediana de 63.5 años. El grupo de pacientes entre 60 y 69 años fue el más numeroso con 52 pacientes, seguido por el grupo de 50-59 años (Tabla 1). El 84.1\% de los consultantes presentaban una DM tipo 2 ( $87.6 \%$ de los hombres y 80.0 $\%$ de las mujeres) (Tabla 1). En el total de 164 pacientes examinados, 32\% resultaron positivos al cultivo (38.5\% en los pacientes DM tipo 1 y el $31 \%$ de los pacientes DM tipo 2). En mujeres, el $23 \%$ presentó un cultivo positivo y en los hombres el $40 \%$ (p 0.015). En los pacientes $<50$ años el $20 \%$ resultó positivo al cultivo, entre los 50 y 69 años el 34 \% y en los de 70 y más años un $53 \%$.

En el total de 202 muestras analizadas, en 63 se obtuvo un cultivo positivo (31\%); de las 34 muestras de pacientes DM tipo 1, 13 de ellas fueron cultivo positivo y en 50 de las 168 muestras de pacientes DM tipo 2 (Tabla 2). Si se analiza por origen de la muestra se observa que el 77\% correspondió a uñas, siendo mayoritaria en DM tipo 1, con una positividad de $37.5 \%$. En 30 muestras de interortejos sólo el 30\% de las muestras fue positiva, sin mayores diferencias entre pacientes DM 1 y DM 2. Sólo 3 muestras plantares DM 1 y 4 DM 2 fueron positivas.

T. rubrum fue siempre mayoritario en los tres tipos de muestras, obteniendo la mayor representatividad en uñas con porcentajes similares (80\%) ya sea en DM 1 y 2 , mientras $T$. mentagrophytes fue escasamente representado, mostrando también en uñas cifras similares en ambos tipos de diabéticos (20\%) (Tabla 3).

\section{DISCUSION}

Los pacientes diabéticos con o sin inmunodepresión asociada o de edad avanzada, presentan una mayor predisposición a padecer infecciones fúngicas superficiales, las cuales varían de leves como onicomicosis, candidiosis oral, balanopostitis a formas clínicas graves con altos índices de morbi y mortalidad. Entre las infecciones fúngicas, destacan tinea pedis y onicomicosis

Tabla 3 Relación de los Dermatofitos aislados según muestra clínica y tipo de diabetes en pacientes con cultivo positivo

\begin{tabular}{|c|c|c|c|c|c|c|c|c|c|c|c|c|c|c|c|c|c|c|c|}
\hline \multirow{4}{*}{ Dermatofito } & \multirow{2}{*}{\multicolumn{5}{|c|}{$\begin{array}{l}\text { MUESTRA DE UÑAS } \\
\text { TIPO DE DIABETES }\end{array}$}} & \multicolumn{6}{|c|}{ MUESTRA INTERORTEJOS } & & \multicolumn{7}{|c|}{ MUESTRAS PLANTARES } \\
\hline & & & & & & \multicolumn{6}{|c|}{ TIPO DE DIABETES } & \multicolumn{8}{|c|}{ TIPO DE DIABETES } \\
\hline & Tipo 1 & \multicolumn{2}{|c|}{ Tipo 2} & \multicolumn{2}{|c|}{ Total } & \multicolumn{2}{|c|}{ Tipo 1} & \multicolumn{2}{|c|}{ Tipo 2} & \multicolumn{2}{|c|}{ Total } & \multicolumn{3}{|c|}{ Tipo1 } & \multicolumn{3}{|c|}{ Tipo 2} & \multicolumn{2}{|c|}{ Total } \\
\hline & $\mathrm{N}^{\circ} \quad \%$ & & & & $\%$ & $\mathbf{N}^{\mathbf{0}}$ & $\%$ & & $\%$ & $\mathbf{N}^{\mathrm{c}}$ & & & $\mathbf{N}^{\mathbf{0}}$ & $\%$ & & & $\%$ & & $\%$ \\
\hline T.rubrum & 780 & 30 & 79 & 37 & 79 & 1 & 100 & & 87.5 & 8 & 8 & & 3 & 100 & & 2 & 50 & 5 & 72 \\
\hline T.mentagrophytes & $2 \quad 20$ & 8 & 21 & 10 & 21 & 0 & 0 & & 12.5 & 1 & 1 & & 0 & 0 & & & 50 & 2 & 28 \\
\hline Total & 9100 & 38 & 100 & & 100 & 1 & 100 & & 100 & 9 & 10 & & 3 & 100 & & & 100 & & 100.0 \\
\hline
\end{tabular}

Asociaciones: 2 pacientes uña y plantar (T. rubrum), 2 paciente uña y interortejo (T. rubrum), 1 paciente interortejo y plantar (T. mentagrophytes) 
que pueden contribuir al agravamiento del pie diabético. Tinea unguium es una de las micosis superficiales más difíciles de tratar y según Roberts \& Evan (1992 ), la presencia de acúmulos hiperqueratósicos presentes en las uñas con gran cantidad de hifas (llamado dermatofitoma subungueal), dificultaría la penetración de antifúngicos. Los agentes involucrados con mayor frecuencia corresponden a Candida spp. y dermatofitos (Principalmente T.rubrum y $T$. mentagrophytes).

Los hallazgos micológicos en los pies de pacientes que concurren a su control de rutina de diabetes, son similares a lo reportado por otros autores. En este estudio, en el $32 \%$ de las muestras clínicas se confirmó una infección fúngica en las diferentes localizaciones de los pacientes diabéticos evaluados, de los cuales el $23 \%$ fueron del sexo femenino y el $40 \%$ del sexo masculino, datos concordantes con publicaciones de otros autores (Lugo-Solominos et al., 1992). En México, Arenas et al. (1999), realizó un estudio micológico de uñas de pies en pacientes ambulatorios con diabetes tipo 2, encontrando onicomicosis en el 31\%, (dermatofitos en un $10.5 \%$ ) con una mayor frecuencia de onicomicosis en diabéticos que en la población general, especialmente en pacientes sin control metabólico adecuado. Por otro lado, Wanzke del Angel et al.(1997), en Brasil encontró un predominio de $21 \%$ en diabéticos ambulatorios mayoritariamente tipo 2, donde la frecuencia de micosis podales por dermatofitos fue la misma que en la población general. López \& Mayorga (2002), comunican una frecuencia mayor (62\%), superior a la encontrada por otros autores, predominando los dermatofitos (T. rubrum y T. mentagrophytes) sobre las levaduras del género Candida.

El porcentaje de aislamiento de T.rubrum es similar a lo comunicado por otros autores. Mayser (2004), realizó un estudio en 95 individuos con DM tipo 1, en $84.6 \%$ se confirmó por examen directo y/ o cultivo, $47.4 \%$ presentaron cultivos positivos y el $69.2 \%$ de los aislamientos correspondió a T.rubrum. Romano et al. (2001) informa que en 7/171 diabéticos ambulatorios se aisló dermatofitos, siendo T.mentagrophytes el agente más frecuentemente aislado y la infección más frecuente fue tinea pedis seguida de onicomicosis subungueal distal. Bouguerra et al. ( 2004 ), en Tunisia, determinó en 307 pacientes diabéticos hospitalizados (tipo 1 y 2), una infección fúngica de pie del 38\%, siendo los dermatofitos los principales responsables (94\%), las localizaciones más frecuentes fueron interdigital (60\%) seguido por onicomicosis (30\%). El principal agente aislado fue T.rubrum.

Mlinaric-Missoni et al. (2005), en 122/509 pacientes diabéticos detectó hongos en pies ( $24 \%$ ), se aislaron 3 especies de dermatofitos Trichophyton rubrum, $\boldsymbol{T}$. mentagrophytes y Epidermophyton. Manzano-Gayosso et al. (1999), reportaron en un estudio comparativo de 60 diabéticos y sus controles que la frecuencia de dermatofitos en micosis superficiales alcanzó un 52\% y 43\%, respectivamente. Por otro lado, García-Humbría et al. (2005), encontraron que los DM tipo 2 presentaron una alta frecuencia de micosis superficiales (75\% diabéticos versus $65 \%$ controles). Ellos presentaron tinea unguium, concomitante con tinea pedis, con una frecuencia más alta. El dermatofito predominante fue T.rubrum, 78\% en diabéticos y 50\% no diabéticos .

Analizando las muestras obtenidas de uñas en nuestro estudio se observó que el 78.7\% correspondió a T. rubrum, y a la vez se confirmó la sospecha clínica de una onicomicosis. Todos los casos diagnosticados correspondieron a una onicomicosis subungueal lateral distal, la forma clínica más frecuentemente observada (dato no mostrado).

Lugo-Somolinos et al. (1992), encontraron que la onicomicosis se presentó en 12 de 31 sujetos diabéticos que presentaron infección micótica. Estos resultados concuerdan con lo reportado por Gupta et al. (1998), que en un estudio multicéntrico de 550 pacientes evaluados, la prevalencia de onicomicosis fue $26 \%$, con una presencia de dermatofitos de $88 \%$, una prevalencia de onicomicosis de un $26 \%$ en pacientes diabéticos (tipo 1 y 2) y que el OR (Odds Ratio) de estos sujetos de padecer esta alteración en la uña es de 2.8 veces más al compararlo con individuos normales (lo que puede atribuirse a que los diabéticos tipo 1 son afectados desde jóvenes), sumado a que los hombres diabéticos del grupo de mayor edad son particularmente propensos a desarrollar infección micótica de la uña.

En India, Dogra et al. (2002), detecta una prevalencia de onicomicosis de un $17 \%$ y el aislamiento de dermatofitos ascendió a un 37\%, con 2.5 veces más probabilidades de tener onicomicosis que los no diabéticos. Saunte et al. (2006), determinó la prevalencia de onicomicosis en pacientes diabéticos de los cuales en 55 casos positivos aisló un 93\% de dermatofitos, con una correlación positiva en los pacientes de mayor edad y con severas alteraciones de las uñas. Analizando la edad, en nuestro estudio, la frecuencia de onicomicosis por dermatofitos a partir de los 60 años fue casi el doble con respecto al grupo etario entre los 40-59 años y en los hombres se presentaron un mayor número de casos. Estos hallazgos concuerdan con Gupta et al. (1998), quienes encontraron que los hombres diabeticos de mayor edad, son particularmente propensos a desarrollar onicomicosis. Además, los hombres tienen hasta 3 veces más posibilidades de tener onicomicosis comparado con las mujeres.

En base a lo expuesto, podemos concluir que tinea unguium y tinea pedis son las más frecuentes en pacientes con DM tipo 2 atendidos en este centro de diabetes y $\boldsymbol{T}$. rubrum es el dermatofito más frecuentemente aislado. Es 
necesario que el paciente y su médico tratante conozcan las posibilidades reales de alcanzar la curación total con un tratamiento sistémico y es muy importante el diagnóstico precoz y tratar adecuadamente esta patología, también cobra importancia la educación en grupos de pacientes con riesgo, brindándoles las recomendaciones para la prevención de onicomicosis y su recidiva en el futuro.

\section{REFERENCIAS}

Arenas, R.; Rubalcaba, P. J.; Leyva, S. J.; Alvarez, Z. B.; San Miguel, F. G.; Ruvalcaba, M.A.; Aranda, P. E. (1999). Onicomicosis y diabetes mellitus tipo 2. Frecuencia en 143 pacientes ambulatorios. Dermatología Rev. Mex. 43:1-7

Bougerra, R.; Essais, O.; Sebai, N.; Ben Salem, L.; Amari, H.; Kammmoun, M.R.; Chaker, E.; Zidi, B.; Ben Slama, C. (2004). Prevalence and clinical aspects of superficial mycosis in hospitalized diabetic patients in Tunisia Med. Mal. Infect. 34:201-5

Dogra, S.; Kumar, B.; Bhansali, A. \& Chakrabarty, A. (2002). Epidemiology of onychomycosis in patients with diabetes mellitus in India. Int. J. Dermatol. 41:647-651

García de los Ríos, M. (1992). Ed. Diabetes Mellitus. Santiago de Chile: Editorial. Arancibia Hnos.

Garcia-Humbría, L.; Richard-Yegres, N.; Pérez-Blanco, M.; Yerres, F.; Mendoza, M.; Acosta, A.; Hernández, R.; Zárraga, E. (2005). Superficial mycoses: comparative study between type 2 diabetic patients and a non diabetic control group. Invest. Clin. 46: 65-64

Gupta, A.K.; Konnikov, N.; MacDonald, P.; Rich, P.; Rodger, N.W.; Edmonds, N.W.; McManus, R.; Summerbell, R.C. (1998). Prevalence and epidemiology of toenail onychomycosis in diabetic subjects: a multicentre survey. Br. J. Dermatol. 139:665-671

Harrison. Principios de Medicina Interna, 2 Vols. (2006). Dennis L. Kasper, Eugene Braunwald, Anthony S. Fauci, Stephen L. Hauser, Dan L. Longo, J. Larry Jameson, y Kurt J. Isselbacher, Eds. Parte 14
Hoog, G.S. de, Guarro, J.; Gene, J. \& Figueras, M.J. (2000). Atlas of Clinical Fungi. 2a. ed. The Netherlands-Spain: CBS/ Universitat Rovira I Virgili.

López, V. \& Mayorga, J. A. (2002). Frecuencia de onicomicosis podal y tiña de los pies en 100 pacientes diabéticos tipo 2 . Dermatología Rev. Mex. 46:254-9

Lugo-Solominos, A. \& Sanchez, J.L. (2002). Prevalence of dermatophytosis in patients with diabetes Journal of the American Academy of Dermatology 26:408-410

Manzano-Gayosso, P.; Mendez-Tovar, L.J. \& López, R. (1995). Frecuencia de micosis superficiales en pacientes diabéticos de consulta externa. Dermatología Rev. Mex. 39:339-342

Mayser, P.; Hensel, J.; Thoma, E.; Podobinska, M.; Geiger, M.; Ulbricht, H.; Haak, T. (2004). Prevalence of fungal foot infections in patients with diabetes mellitus type 1- underestimation of moccasin type tinea. Exp. Clin. Endocrinol. Diabetes 112:264-8

Mlinariæ-Missoni, E.; Kaleniæ, S.; Vaziæ-Babiæ, V. (2005). Species distribution and frequency of isolation of yeasts and dermatophytes from toe webs of diabetic patients. 13:85-92

Romano, C.; Massai, L.; Asta, F.; Signorini, A.M. (2001). Prevalence of dermatophytic skin and nail infections in diabetic patients. Mycoses 44:83-86

Roberts, D.T. \& Evans, G. (1992). Prevalence of dermatophyte onychomycosis in the United Kingdom: Results of an omnibus survey. Br. J. Dermatology; 126:S23-S27

Saunte, D.M.; Holgersen, J.B.; Haedersdal, M.; Strauss, G.; Bitsch, M.; Svendsen, O.L.; Arendrup, M.C.; Svejgaard, E.L. (2006). Prevalence of toe nail onychomicosis in diabetic patients. Acta Derm. Venereol. 86:425-8

Wanzke del Angel, V.; Arce, M.; Arenas, R.; Trejo-Izquierdo, E.; Rocha-Reyes, M.; Miranda-Garduño, L.M.; Fabián San Miguel, G. (1997). Detección de micosis podales y portadores en pacientes diabéticos ambulatorios. Estudio clínico micológico en 106 pacientes. Dermatologia Rev. Mex. 41:216-222 